\section{Natural Products Research}

\section{Kerwin Sean M}

Medicinal chemistry, College of Pharmacy, The University of Texas, Austin, USA

Alternatively maligned and championed by pharmaceutical companies and biomedical research funding agencies, natural products research has and will continue to enable life-saving therapies. But what will be the future of natural products research look like and how will we get there? The launch of Natural Products Chemistry and Research presents an occasion to contemplate the frontiers of the discipline and look forward to what may lie ahead. In this note, current trends and potential new developments are discussed. Some aspects of current research trends will certainly continue to drive the field. Bioassayguided isolation will continue to provide new natural products with fascinating structures and promising activities. With regard to the latter, there will be growing emphasis on more information-rich activity screens in place of standard cytotoxicity or antimicrobial assays, to better differentiate truly promising and selective biologically active natural products. Chemical ecology will continue to impact drug discovery efforts, with growing attention to processes such as quorum sensing and other inter- and intra-species chemical communication pathways. Synthetic studies of natural products will continue to be the gold standard by which synthetic strategies, tactics, and methods are tested, but will place an even greater emphasis on efficiency and practicality. In addition, synthetic approaches that focus on affording analogs designed to maximize target identification and optimization by SAR will continue to be emphasized, and the use of natural products as starting materials for the elaboration of libraries will continue to mimic the way nature accesses diversity through the multifold processing of intermediate secondary metabolites. Finally, the studies of new and previously identified natural products by ever more sophisticated biochemical, cellular, and whole-animal approaches will continue to shed light on complex biological processes and provide leads for drug discovery.

There are also new trends in natural products research that promise to have a profound impact on the field. The growing trend of "designer" natural products produced through re-engineering biosynthetic pathways will both test our understanding of secondary metabolism and provide novel compounds with unique activities. In addition, there will be a growing emphasis on identifying biosynthetic pathways and natural products directly from "environmental" DNA. Polypharmacy and polypharmacology present particular challenges and opportunities in studies of natural products-based alternative medicines and in the discovery of new therapeutics. New approaches in systems biology and studies of signaling pathways will enable the characterization of the effects of natural products as single compounds or as mixtures on entire pathways and cross-talk between pathways. Finally, the nexus between combinatorial chemistry and natural products chemistry will provide additional tools to evaluate the chemical space that is occupied by natural products and to exploit this in the design of higher quality, natural product-like libraries for the identification of molecular probes and hits for drug discovery.

The scope and impact of natural products research will continue to expand. This provides an opportunity for new forums for the dissemination of high-quality work in this field. It is believed that Natural Products Chemistry and Research will provide such a forum for publications in all of these established and evolving areas of natural products research.
*Corresponding author: Kerwin Sean M, Associate Professor of Medicinal Chemistry, College of Pharmacy, The University of Texas, Austin, USA, Tel: 512471-5074; E-mail: skerwin@austin.utexas.edu

Received October 26, 2012; Accepted October 27, 2012; Published October 29 2012

Citation: KSean M (2012) Natural Products Research. Nat Prod Chem Res 1:e101. doi:10.4172/2329-6836.1000e101

Copyright: @ 2012 Kerwin Sean M. This is an open-access article distributed under the terms of the Creative Commons Attribution License, which permits unrestricted use, distribution, and reproduction in any medium, provided the original author and source are credited. 